\title{
Antitumour Effects of Somatostatin Analogues in the Treatment of Neuroendocrine Tumours
}

\author{
Diogo Assed Bastos, ${ }^{1}$ Brenda Gumz ${ }^{2}$ and Frederico Costa ${ }^{3}$
}

1. Hematologist; 2. Physician; 3. Medical Oncologist, Oncology Center, Hospital Sírio-Libanês, São Paulo, Brazil

\begin{abstract}
Neuroendocrine tumours (NETS) are rare and heterogeneous neoplasms that can present with functional syndromes due to the hypersecretion of peptides. Somatostatin analogues (SSAS) have been used since the 1980s for the treatment of neuroendocrine tumours, with the aim of controlling the symptoms of functioning tumours and improving patients' quality of life. Data from preclinical studies offer evidence of both direct and indirect mechanisms by which SSAs can exert antitumour effects. The activation of somatostatin receptors by SSAs leads to the activation of phosphotyrosine phosphatases, which control downstream apoptotic and antiproliferation signalling pathways. Also, the suppression of secretion of several growth factors and inhibition of antiangiogenic activity by SSAS indirectly inhibits tumour cell proliferation in vitro. Previous uncontrolled studies had shown tumour shrinkage and disappearance in response to the SSA octreotide. Recent results from the randomised and placebo-controlled PROMID trial have demonstrated that octreotide has antitumour activity in patients with metastatic mid-gut NETs. This article examines recent data providing evidence of the antitumour activity of somatostatin analogues.
\end{abstract}

\section{Keywords}

Neuroendocrine tumour (NET), somatostatin, antiproliferative activity, octreotide long-acting release (LAR), lanreotide

Disclosure: The authors have no conflicts of interest to declare.

Acknowledgement(s): Editorial assistance was provided by Janet Manson at Touch Briefings.

Received: 26 June 2012 Accepted: 5 July 2012 Citation: European Oncology \& Haematology, 2012;8(3):156-60 DOI: 10.17925/EOH.2012.08.3.156

Correspondence: Frederico Costa, Oncology Center, Hospital Sírio-Libanês, Adma Jafet Street, 91, São Paulo, SP, 0138-050, Brazil. E: Frederico.costa@hsl.org.br

Support: The publication of this article was funded by Novartis. The views and opinions expressed are those of the authors and not necessarily those of Novartis.

Neuroendocrine tumours (NETS) are rare neoplasms that arise from neuroendocrine cells which are present throughout the body. NETS may be classified as functioning or non-functioning and are further differentiated based on the site of primary origin, histologic grade (low, intermediate or high) and proliferation rate. ${ }^{1}$ Functioning NETS are characterised by excessive hormone production and release, and cause hormonal syndromes (outlined in Table 1), while non-functioning NETs are not associated with a distinct hormonal syndrome - although they can secrete hormones and peptides at subclinical levels. Approximately $60-70 \%$ of NET patients are diagnosed with disseminated metastatic disease. ${ }^{2}$ Even though many patients have indolent disease, some patients present with overtly aggressive tumours. Due to this heterogeneity, treatment is often individualised and may involve several modalities, such as cytoreductive surgery, non-surgical liver-directed therapies, somatostatin analogues, chemotherapy and, more recently, targeted agents. $^{3}$ These treatments are used with two major objectives: to control the symptoms of hormone hypersecretion and to control tumour growth. The aim of this article is to review the antitumour effects of somatostatin (STS) analogues in the treatment of NETS.

\section{Somatostatin}

STS is a peptide produced by intestinal paracrine cells and plays a major role in inhibition of gastrointestinal (GI) endocrine secretion. ${ }^{4,5}$
STS acts as a paracrine, autocrine or neuronal regulatory molecule to inhibit glandular secretion, neurotransmission, smooth muscle contractility and absorption of nutrients, regulating the functioning of activated immune cells. ${ }^{5-7}$ STS exerts control over several neurophysiological functions, and is a pan-inhibitory agent for all known GI tract hormones. ${ }^{8}$ In the GI tract, STS promotes reduction of liver and splanchnic blood flow, inhibits Gl and pancreatic secretions, inhibits gallbladder contractility and bile flow, slows GI transit, inhibits absorption of glucose and amino acids and inhibits tissue growth and proliferation. ${ }^{5}$

STS is a cyclic peptide. Its two biological molecular forms are somatostatin-14 (S-14) and somatostatin-28 (S-28), which are both expressed throughout the Gl tract. STS is synthesised as part of a large precursor molecule that is cleaved, enzymatically processed and released by endocrine and nerve cells. ${ }^{9}$ STS binds to five different STS receptor subtypes (numbered 1-5) present at the surface of different endocrine cell types.

\section{Somatostatin Analogues}

Initially, the clinical use of native STS was limited because of the very short half-life of the peptide (less than three minutes in the circulation) and the need for continuous intravenous administration. As a result, formulations of somatostatin analogues (SSAS) with 
Table 1. Clinical Features of Functioning Neuroendocrine Tumours

\begin{tabular}{lll}
$\begin{array}{ll}\text { Tumour Type } \\
\text { Insulinoma }\end{array}$ & Hormone Produced & Symptoms \\
\hline Gastrinoma & Insulin, proinsulin & Hypoglycaemia, confusion, sweating, dizziness, weakness, coma, relief with eating \\
\hline VIPoma & Gastrin & Severe peptic ulceration and diarrhoea (Zollinger-Ellison syndrome) \\
\hline PPoma & Vasointestinal peptide (VIP) & Profuse watery diarrhoea, hypokalaemia, achlorhydria (Verner-Morrison syndrome) \\
\hline Somatostatinoma & Pancreatic peptide (PP) & Weight loss, jaundice, abdominal pain \\
\hline Glucagonoma & Somatostatin & Gallstones, weight loss, diarrhoea, diabetes \\
\hline
\end{tabular}

Source: reprinted from Clinical Oncology, vol 24, KE Oberg, The Management of Neuroendocrine Tumours: Current and Future Medical Therapy Options, 282-93, ${ }^{3}$ copyright (2012) with permission from the Royal College of Radiologists.

\section{Table 2: Characteristics of the Five Somatostatin Receptor Subtypes}

\begin{tabular}{|c|c|c|c|c|c|}
\hline & \multicolumn{5}{|c|}{ Somatostatin Receptor Subtype } \\
\hline & 1 & 2 & 3 & 4 & 5 \\
\hline Position on the chromosome & $14 q 13$ & $17 q 24$ & $22 q 13.1$ & 20p11.2 & $16 p 13.3$ \\
\hline \multicolumn{6}{|c|}{ Receptor-binding affinity $\left(\mathrm{IC}_{50}\right.$ in $\left.\mathrm{mol} / \mathrm{l}\right)$ : } \\
\hline - Somatostatin ${ }^{14}$ & 0.93 & 0.15 & 0.56 & 1.50 & 0.29 \\
\hline - Octreotide $^{14}$ & 280.00 & 0.38 & 7.10 & $>1,000$ & 6.30 \\
\hline - Lanreotide $^{15}$ & $>1,000$ & 0.80 & 107 & $>1,000$ & 5.20 \\
\hline Presence in normal tissues & $\begin{array}{l}\text { Brain, lungs, stomach, } \\
\text { jejunum, kidneys, } \\
\text { liver, pancreas }\end{array}$ & Brain, kidneys & Brain, pancreas & Brain, lungs & $\begin{array}{l}\text { Brain, heart, adrenals, } \\
\text { placenta, pituitary, small } \\
\text { intestine, skeletal muscle }\end{array}$ \\
\hline Induction of G1 cell cycle arrest & Yes & Yes & - & Yes & Yes \\
\hline Induction of apoptosis & - & Yes & Yes & - & - \\
\hline \multicolumn{6}{|l|}{ Distribution in GEP-NET (\%): ${ }^{10,11,51}$} \\
\hline - Non-functioning tumour & 80 & 100 & 40 & 100 & 60 \\
\hline - Gastrinoma & 33 & 50 & 17 & 83 & 50 \\
\hline - Glucagonoma & 67 & 100 & 67 & 67 & 67 \\
\hline - VIPoma & 100 & 100 & 100 & 100 & 100 \\
\hline - All & 68 & 86 & 46 & 93 & 57 \\
\hline
\end{tabular}

$I_{50}=$ half maximal inhibitory concentration (i.e., concentration required for a $50 \%$ inhibition of the binding somatostatin or somatostatin analogue to the receptor subtype); GEP-NET = gastroenteropancreatic neuroendocrine tumour; VIP = vasointestinal peptide. Source: adapted from Lamberts et al., 1996 and Strosberg and Kvols, 2010.13

longer half-lives have been developed. First, short-acting analogues with 6-8 hour half-lives - such as octreotide (SMS201-995), vapreotide (RC-160), lanreotide (BIM 23014) and seglitide (MK 678) became available. Later, long-acting formulations of octreotide (octreotide long-acting release [LAR]) and of lanreotide (slow-release [SR] lanreotide), with half-lives of almost 28 days, were developed..$^{10,11}$ Since the 1980s, SSAs have been the standard of care for NET symptom control, ${ }^{12}$ and they have well documented efficacy in controlling functioning tumour symptoms. ${ }^{2,11}$ Adverse events associated with SSAs are usually mild and include nausea, abdominal discomfort, bloating, fat malabsorption and gallstone formation. ${ }^{5}$

Although native STS binds all the five receptor subtypes, the synthetic analogues have different affinities for the STS receptors and exert their actions mainly interacting with subtypes 2 and 5 (see Table 2). , $13-15^{-1}$ More recently, pasireotide, a new SSA, has been developed and this molecule demonstrates higher affinity for subtypes 1, 2, 3 and 5. 14,16,17

\section{Somatostatin Receptors}

The STS receptor is a G protein-coupled receptor present in the membranes of cells from various organs such as the endocrine and exocrine pancreas, GI tract, anterior pituitary and various regions of the brain..$^{18}$ There are five known STS receptor subtypes, and these subtypes may have different expression levels and functions depending on their location throughout the body (see Table 2).
Although these receptors are similar in their binding to S-14 and S-28, the binding affinity of each subtype is different for the SSAS. ${ }^{19,20}$

All STS receptor subtypes are coupled to adenylate cyclase through an inhibitory $G$ protein, causing a reduction in intracellular cyclic adenosine monophosphate (CAMP) concentration when activated.? Furthermore, activation of the receptor may result in ion channel activation, decreasing the influx of calcium to the cell. ${ }^{5}$ There is evidence that the STS receptors can change ligand-binding affinity and receptor internalisation through agonist-induced dimerisation. ${ }^{21}$ Additionally, STS receptors can interact and form heterodimers with other families of receptors, and thus alter receptor signalling and provide novel molecular pathways for cell proliferation and survival. ${ }^{21-23}$

\section{Antiproliferative Mechanisms}

Based on many different in vitro and in vivo experimental models, the antitumour activity of SSAs has been demonstrated and is mediated through direct and indirect mechanisms. ${ }^{22,24-26}$ The activation of STS receptors can cause cytostatic and pro-apoptotic effects by direct activation of phosphotyrosine phosphatases (PTPS). ${ }^{27,28}$ PTPS control downstream signalling molecules such as the mitogen-activated proteins (MAPS) extracellular signal-regulated kinase (ERK)1 and ERK2, and ultimately induce antiproliferation by upregulation of cyclin-dependent kinase inhibitors.22 The STS receptor subtypes 2 and 3 are involved with the pro-apoptotic mechanisms through the 
Table 3: Antiproliferative Activity and Principal Transducing Systems Regulated by Somatostatin Receptors According to Subtype

\begin{tabular}{|c|c|c|c|c|c|}
\hline & \multicolumn{5}{|c|}{ Somatostatin Receptor Subtype } \\
\hline & 1 & 2 & 3 & 4 & 5 \\
\hline \multicolumn{6}{|l|}{ Antiproliferative Activity } \\
\hline Cell cycle progression & $\downarrow$ & $\downarrow$ & $\downarrow$ & $\uparrow \downarrow$ & $\downarrow$ \\
\hline Apoptosis & - & $\uparrow$ & $\uparrow$ & - & - \\
\hline \multicolumn{6}{|l|}{ CAMP Production } \\
\hline Adenylyl cyclase & $\downarrow$ & $\downarrow$ & $\downarrow$ & $\downarrow$ & $\downarrow$ \\
\hline \multicolumn{6}{|l|}{ Ion Currents } \\
\hline Voltage-sensitive $\mathrm{Ca}++$ & $\downarrow$ & $\downarrow$ & - & - & $\downarrow$ \\
\hline K+ current & $\uparrow$ & $\uparrow$ & $\uparrow$ & $\uparrow$ & $\uparrow$ \\
\hline GIRK activity & - & 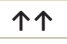 & $\uparrow$ & $\uparrow$ & $\uparrow$ \\
\hline \multicolumn{6}{|l|}{ MAP Kinases } \\
\hline ERK 1 and 2 & $\downarrow \uparrow$ & $\downarrow \uparrow$ & $\downarrow$ & $\uparrow$ & $\downarrow$ \\
\hline p38 & & $\uparrow$ & - & $\uparrow$ & \\
\hline JNK & - & $\downarrow$ & - & - & $\uparrow$ \\
\hline \multicolumn{6}{|l|}{ Tyrosine Kinases } \\
\hline SrC & $\uparrow$ & $\uparrow$ & & & \\
\hline Jak2 & $\uparrow$ & $\uparrow$ & & & \\
\hline \multicolumn{6}{|l|}{ Phospholipid Kinase } \\
\hline PI3K & $\downarrow \uparrow$ & $\downarrow \uparrow$ & & & \\
\hline \multicolumn{6}{|l|}{ Nitric Oxide Synthases } \\
\hline nNOS & - & $\uparrow$ & & & $\downarrow$ \\
\hline eNOS & $\downarrow$ & $\downarrow$ & $\downarrow$ & - & \\
\hline \multicolumn{6}{|l|}{$\mathrm{Na}+/ \mathrm{H}+$ Exchanger } \\
\hline NEH1 & $\downarrow$ & $\downarrow \uparrow$ & - & $\uparrow$ & - \\
\hline
\end{tabular}

$\uparrow=$ activation; $\downarrow$ = inhibition; - = no effect; $C a++=$ calcium; $c$ AMP = cyclic adenosine monophosphate; eNOS = endothelial nitric oxide synthase; $E R K=$ extracellular signal-regulated kinase; GIRK = G protein-gated K+ channel; Jak2 = Janus kinase 2; JNK = C-Jun N-terminal kinase; $M A P=$ mitogen-activated protein; $N E H 1=$ sodium/hydrogen exchanger 1; nNOS = neuronal nitric oxide synthase; PI3K = phosphatidylinositol 3-kinase; Src $=$ proto-oncogene tyrosine-protein kinase.

Source: republished with permission of Frontiers in Bioscience from Molecular mechanisms of the antiproliferative activity of somatostatin receptors (SSTRS) in neuroendocrine tumors,

T Florio, vol 13, 2008); 22 permission conveyed through Copyright Clearance Center, Inc.

activation of pro-apoptotic genes such as p53 and bcl-2 (subtype 3), ${ }^{29}$ the inhibition of the antiapoptotic effects of MAP kinase (subtype 2$)^{30}$ and the sensitisation of death receptors (subtypes 2 and 3 ). ${ }^{31}$ In addition to direct effects, SST and SSAS exert a number of indirect antiproliferative, antiangiogenic and immunomodulatory effects. . $3,20,32^{2}$ These include: inhibition of the release of hormones and growth factors that aid tumour growth, such as insulin-like growth factor (IGF) and vascular endothelial growth factor (VEGF); reduction of tumour blood flow due to the suppression of endothelial cell proliferation; and arrest of monocyte migration (see Table 3).

\section{Results from Previous Trials and Case Reports}

More than 30 cases of partial tumour regression following octreotide administration, with or without other therapies, have been reported in the literature..$^{33,34}$ Octreotide treatment of a patient with metastatic vasointestinal peptide (VIP)oma induced a reduction in the number and size of liver secondary tumours, as evidenced by computerised axial tomography, ${ }^{35}$ and a similar finding has been reported in a patient with gastrinoma. ${ }^{36}$ In addition, several case reports have documented complete histological regression of metastatic tumours after treatment with octreotide. ${ }^{33,37,38}$

In addition to these case studies, several clinical studies have shown results suggesting that octreotide and lanreotide have antitumour activity in patients with progressive NETs. ${ }^{39-46}$ In a prospective study of 103 patients with metastatic NETs, $37 \%$ of patients who had confirmed tumour progression experienced stabilisation for at least three months. ${ }^{40}$ In the patient group who had stable disease prior to octreotide treatment, $54 \%$ continued to have stable disease with no progression for over 12 months. A Phase II trial of SR lanreotide (30 mg) in 55 patients found that, of 31 assessable patients, 25 (81\%) achieved disease stability, ${ }^{46}$ while a second Phase II trial comprising 39 evaluable patients found that 19 (49\%) experienced stable disease. ${ }^{42}$ The administration of octreotide or lanreotide in regular doses has been demonstrated to induce stabilisation of tumour growth in 45.2-52.0\% and $32-81 \%$ of patients, respectively. ${ }^{41-46}$ Unfortunately, the studies included heterogeneous tumour types, none was placebo-controlled and none evaluated only treatment-naive patients. The 2009 PROMID (Placebo-controlled, double-blind, prospective, randomized study on the effect of octreotide LAR in the control of tumor growth in patients with metastatic neuroendocrine midgut tumors) study was designed to address these shortcomings.

\section{Results from the PROMID Study}

PROMID was a randomised, double-blind, placebo-controlled trial conducted in 18 German academic centres. ${ }^{47} \mathrm{~A}$ total of 85 patients with locally inoperable or metastatic well-differentiated mid-gut NETS or tumours of unknown primary origin (but believed to be of mid-gut origin following exclusion of the presence of primary tumour within the pancreas or chest) were randomly assigned 1:1 to octreotide LAR $30 \mathrm{mg}$ or placebo until progression. The primary endpoint was time to progression (TTP) assessed by computed tomography or magnetic resonance imaging.

Patients were highly selected and their cases discussed at the local institutional tumour boards prior to inclusion. Only previously untreated patients not eligible for curative-intended surgery and able to be observed without intolerable symptoms were included. The baseline characteristics of the 1:1 random assignment of the two study arms were well balanced for study centre, presence of metastases, tumour functionality, Ki-67 index and age. Among the 85 patients, $58 \%$ had non-functioning tumours and $23 \%$ had tumours of unknown primary origin. The hepatic tumour burden was less than $10 \%$ in $75 \%$ of all patients. Patients were randomised to octreotide LAR $(n=42)$ or placebo $(n=43)$. Prior to tumour progression, seven patients withdrew treatment consent (five from the octreotide LAR arm and two from the placebo arm), one patient switched from the placebo arm to the octreotide LAR arm and five patients withdrew from the octreotide LAR arm due to adverse events. Hence there were 33 patients in the octreotide LAR arm and 40 patients in the placebo arm.

After six months, a significant difference was seen in the numbers of patients who had stable disease $(66.7 \%$ in the octreotide LAR arm versus $37.2 \%$ in the placebo arm). The confirmatory interim analysis observed 26 and 40 progressions in the octreotide LAR and placebo arms, respectively (hazard ratio [HR] $=0.34 ; 95 \%$ confidence interval [Cl] $0.20-0.59 ; \quad p=0.000072$ ) (see Figure 1). Octreotide greatly improved the TTP: the median TTP was 14.3 and 6.0 months in the octreotide LAR and placebo arms, respectively $(\mathrm{HR}=0.34)$. This study thus provided evidence that octreotide LAR inhibits tumour proliferation in patients with well-differentiated metastatic mid-gut NETs.

The major criticism for this study was the lack of progression status prior to accrual. One of the great advantages of a prospective 
randomised study is the understanding of tumour natural history in the placebo arm. In the placebo arm, $53 \%$ of patients had progressed by WHO criteria at six months; $79 \%$ had progressed at 12 months; and almost all had progressed at two years. The use of octreotide LAR demonstrated a clear antiproliferative effect. In the treatment arm, 29 patients had disease control (one partial remission [PR], 28 stable disease [SD]) versus 17 patients in the placebo arm (one PR, 16 SD) $(p=0079)$. As mentioned previously, the median TTP was 14.3 months for octreotide LAR and 6.0 months for placebo. The difference in tumour progression between the arms was 8.3 months. This is a robust and significant result, but is the benefit clinically meaningful?

The expected survival for a low-burden asymptomatic mid-gut NET is over seven years. Given this timeframe, the 8.3 month increase in TTP in the octreotide LAR arm does not initially seem a significant improvement; however, when studying the survival curve in the treatment arm closely, it becomes apparent that 16 patients had no progression and a long-lasting response for many years. Octreotide LAR appears to be a truly targeted agent, and a durable response in a third of the patient population is definitely clinically meaningful. A lowtoxicity intervention that induces a durable response and does not preclude future new interventions is the optimal treatment strategy for improving prognosis in NET patients. This is the main reason why patients with well-differentiated mid-gut NET should be offered SSAS independent of their tumour progression status or positivity on octreotide scan (Octreoscan ${ }^{\mathrm{TM}}$ ). SSAs are now accepted as the new standard treatment for patients with low-grade mid-gut NET.

In the PROMID study, median overall survival had not been reached in the treatment arm after 77.4 months of follow-up. In the placebo arm, the median overall survival was 73.7 months; however, this estimation was not robust due to the low number of deaths $(p=0.77)$. since cross-over could occur after the trial had been unblinded, $77 \%$ of the patients in the placebo arm received octreotide LAR, and additionally some patients in the placebo arm were treated with hepatic chemoembolisation, peptide receptor radionuclear therapy and chemotherapy. These therapeutic interventions may have had an undetermined effect, making the impact of octreotide LAR on patient survival difficult to assess.

\section{Future Directions for Somatostatin Analogues}

Results from the PROMID trial have proven the benefit of octreotide LAR treatment for patients with mid-gut NETs. The ongoing Controlled study of lanreotide antiproliferative response in NET (CLARINET) trial has been designed to examine the efficacy of lanreotide (autogel $120 \mathrm{mg}$ ) in the treatment of NETs. This trial is designed to assess whether lanreotide autogel prolongs the time to disease progression in patients with non-functioning NETs, including those of pancreatic origin. ${ }^{48}$ Additionally, the activity of pasireotide as an antitumour agent is currently being investigated in a Phase II trial, whose goal is to determine whether pasireotide LAR can shrink or slow the growth of metastatic neuroendocrine carcinomas. ${ }^{49}$

\section{Figure 1: Confirmatory Intention-to-treat Analysis of Time to Progression or Tumour-related Death}

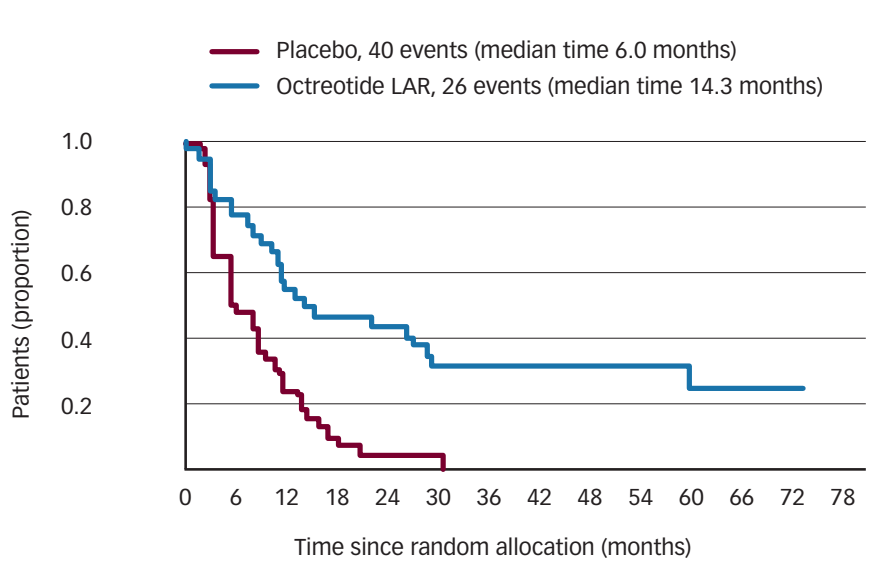

No. of patients at risk

$\begin{array}{lllllllllllllll}\text { Placebo } & 43 & 21 & 9 & 3 & 1 & 1 & 0 & 0 & 0 & 0 & 0 & 0 & 0 & 0\end{array}$ $\begin{array}{llllllllllllll}\text { Octreotide LAR } 42 & 30 & 19 & 16 & 15 & 10 & 10 & 9 & 9 & 6 & 5 & 3 & 1 & 0\end{array}$

Log-rank test stratified by functional activity: $\mathrm{p}=0.000072, \mathrm{HR}=0.34(95 \% \mathrm{Cl} 0.20-0.59)$

$\mathrm{Cl}=$ confidence interval; $H R=$ hazard ratio; $L A R=$ long-acting release . Source: Rinke et al., 2009.7?

Although SSAs are the first-line therapy for mid-gut NETs, the combination of these agents with other therapies may be needed in order to improve clinical outcomes. The potential synergy of the mammalian target of rapamycin (mTOR) inhibitor everolimus with octreotide was recently assessed in the Phase III RAD001 in advanced neuroendocrine tumours (RADIANT)-2 trial..$^{50}$ Treatment with octreotide plus everolimus improved progression-free survival in patients with advanced NETs compared with octreotide plus placebo (16.4 months versus 11.3 months, respectively).

\section{Conclusion}

The antiproliferative effect of the SSA octreotide LAR in NET patients was confirmed in the PROMID study, in which treatment with octreotide LAR greatly improved the TTP (14.3 versus 6.0 months in the placebo arm; HR=0.34). It is important to select patients with lowor intermediate-grade NETS with good prognosis or slow-growing disease to undergo this antiproliferative treatment strategy. NET patients with more aggressive and poorly differentiated tumours - a population not included in the PROMID study - should be treated by other means. The SSA-mediated benefit of octreotide LAR in terms of TTP is augmented by the long-lasting tumour control in a significant proportion of patients with indolent disease. This durable response is a clinically meaningful benefit of a low-toxicity treatment strategy that does not preclude future treatment interventions. Preclinical data demonstrate that SSAS have antitumour activity and this is mediated by both direct and indirect effects. These effects serve as the basis for developing new treatment combinations for NET patients that include SSAs as antiproliferative drugs and not just for symptomatic control.
1. Oberg K, Castellano D, Current knowledge on diagnosis and staging of neuroendocrine tumors, Cancer Metastasis Rev , 2011;30(Suppl. 1):3-7.

2. Modlin IM, Oberg K, Chung DC, et al., Gastroenteropancreatic neuroendocrine tumours, Lancet Oncol, 2008:9:61-72.

3. Oberg KE, The management of neuroendocrine tumours: current and future medical therapy options, Clin Oncol (R Coll Radiol), 2012;24:282-93.

4. Brazeau P, Vale W, Burgus R, et al., Hypothalamic polypeptide that inhibits the secretion of immunoreactive pituitary growth hormone, Science, 1973;179:77-9.

5. Lamberts SW, van der Lely AJ, de Herder WW, Hofland LJ, Octreotide, N Engl J Med, 1996;334:246-54

6. Reichlin S, Somatostatin, N Engl I Med, 1983:309:1495-501.

7. Reichlin $S$, Neuroendocrine-immune interactions, N Engl I Med, 1993;329:1246-53.

N Eng/ I Med, 1993;329:1246-53.
8. Reichlin S, Secretion of somatostatin and its physiologic function, J Lab Clin Med, 1987;109:320-6.

9. Patel YC, Somatostatin and its receptor family,
Front Neuroendocrinol, 1999;20:157-98.

10. Appetecchia $M$, Baldelli $R$, Somatostatin analogues in the treatment of gastroenteropancreatic neuroendocrine tumours, current aspects and new perspectives, J Exp Clin Cancer Res, 2010:29:19.

11. Oberg K, Management of neuroendocrine tumours, Ann Oncol, 2004;15(Suppl. 4):iv293-8.

12. Kvols LK, Moertel CG, O'Connell MJ, et al., Treatment of the malignant carcinoid syndrome. Evaluation of a long-acting somatostatin analogue, N Engl J Med, 1986;315:663-6. 
13. Strosberg J, Kvols L, Antiproliferative effect of somatostatin analogs in gastroenteropancreatic neuroendocrine tumors, World I Gastroenterol, 2010;16:2963-70.

14. Bruns C, Lewis I, Briner U, et al., SOM230: a novel somatostatin peptidomimetic with broad somatotropin release inhibiting factor (SRIF) receptor binding and a unique antisecretory profile, Eur J Endocrinol, 2002:146:707-16.

15. Hofland $\mathrm{L}$, Lamberts SW, The pathophysiological consequences of somatostatin receptor internalization and resistance, Endocr Rev, 2003;24:28-47.

16. Schmid HA, Pasireotide (SOM230): development, mechanism of action and potential applications, Mol Cell Endocrinol, 2008;286:69-74.

17. Schmid HA, Silva A, Pasireotide (SOM230) a new somatostatin analogue for the treatment of acromegaly, Cushing's disease and neuroendocrine tumors, Acta Physiologica, 2009:195(Suppl. 669):Abstract P458.

18. Reubi JC, Kvols L, Krenning E, Lamberts SW, Distribution of somatostatin receptors in normal and tumor tissue, Metabolism, 1990;39(9 Suppl. 2):78-81.

19. Kidd M, Drozdov I, Joseph R, et al., Differential cytotoxicity of novel somatostatin and dopamine chimeric compounds on bronchopulmonary and small intestinal neuroendocrine tumor cell lines, Cancer, 2008;113:690-700.

20. Susini $C$, Buscail $L$, Rationale for the use of somatostatin analogs as antitumor agents, Ann Oncol, 2006;17:1733-42.

21. Rocheville M, Lange DC, Kumar U, et al., Subtypes of the somatostatin receptor assemble as functional homo- and heterodimers, J Biol Chem, 2000:275:7862-9.

22. Florio T, Molecular mechanisms of the antiproliferative activity of somatostatin receptors (SSTRS) in neuroendocrin tumors, Front Biosci, 2008;13:822-40.

23. Kumar U, Cross-talk and modulation of signaling between somatostatin and growth factor receptors, Endocrine, 2011;40:168-80

24. Grozinsky-Glasberg S, Franchi G, Teng M, et al., Octreotide and the MTOR inhibitor RAD001 (everolimus) block proliferation and interact with the Akt-mTOR-p70S6K pathway in a neuro-endocrine tumour cell line, pathway in a neuro-endocrine tumour
Neuroendocrinology, 2008;87:168-81.

25. Tsagarakis NJ, Drygiannakis I, Batistakis AG, et al., Octreotide induces caspase activation and apoptosis in human hepatoma HepG2 cells, World I Gastroenterol, 2011;17:313-21.

26. Ferrante $\mathrm{E}$, Pellegrini $\mathrm{C}$, Bondioni S, et al., Octreotide promotes apoptosis in human somatotroph tumor cells by activating somatostatin receptor type 2, Endocr Relat Cancer, 2006;13:955-62.

27. Florio T, Somatostatin/somatostatin receptor signalling: phosphotyrosine phosphatases, Mol Cell Endocrinol,
2008;286:40-8

28. Florio T, Thellung S, Arena S, et al., Somatostatin receptor (SSTR1)-mediated inhibition of cell proliferation correlates with the activation of the MAP kinase cascade: role of the phosphotyrosine phosphatase SHP-2, J Physiol Paris, 2000:94:239-50.

29. Sharma $\mathrm{K}$ srikant $\mathrm{CB}$, Induction of wild-type $\mathrm{p} 53$, Bax, and acidic endonuclease during somatostatin-signaled apoptosis in MCF-7 human breast cancer cells, Int I Cancer, 1998;76:259-66.

30. Guillermet-Guibert J, Saint-Laurent N, Davenne L, et al Novel synergistic mechanism for sst2 somatostatin an TNFalpha receptors to induce apoptosis: crosstalk between NF-kappaB and JNK pathways, Cell Death Differ, 2007;14:197-208

31. Guillermet J, Saint-Laurent $N$, Rochaix $P$, et al., Somatostatin receptor subtype 2 sensitizes human pancreatic cancer cells to death ligand-induced apoptosis, Proc Natl Acad Sci U S A, 2003;100:155-60.

32. Grozinsky-Glasberg S, Shimon I, Korbonits M, Grossman AB, Somatostatin analogues in the control of neuroendocrine tumours: efficacy and mechanisms, Endocr Relat Cancer, 2008;15:701-20.

33. Leong WL, Pasieka JL, Regression of metastatic carcinoid tumors with octreotide therapy: two case reports and a review of the literature, I Surg Oncol, 2002;79:180-7.

34. Yamashita K, Takase S, Nakamura T, et al., [A case of recta carcinoid with multiple liver, lymph nodes and bone metastases that responded to an octreotide therapyl, Gan To Kagaku Ryoho, 2010;37:2349-51.

35. Clements $D$, Elias E, Regression of metastatic vipoma with somatostatin analogue SMS 201-995, Lancet, 1985;1:874-5.

36. Shepherd JJ, Senator GB, Regression of liver metastases in patient with gastrin-secreting tumour treated with SMS 201-995, Lancet, 1986;2:574.

37. Imtiaz KE, Monteith P, Khaleeli A, Complete histological regression of metastatic carcinoid tumour after treatment with octreotide, Clin Endocrinol (Oxf), 2000:53:755-8.

38. Wadamori K, Oka M, Shimizu R, et al., [A case of multiple liver metastasis from ileac carcinoid effectively treated with continuous intraarterial infusion of somatostatin analog] Gan To Kagaku Ryoho, 1995;22:1669-72.

39. Arnold R, Rinke A, Klose KJ, et al., Octreotide versus octreotide plus interferon-alpha in endocrine gastroenteropancreatic tumors: a randomized trial, Clin Gastroenterol Hepatol, 2005;3:761-71.

40. Arnold R, Trautmann ME, Creutzfeldt W, et al., Somatostatin analogue octreotide and inhibition of tumour growth in metastatic endocrine gastroenteropancreatic tumours, Gut, 1996;38:430-8.
41. Di Bartolomeo M, Bajetta E, Buzzoni R, et al., Clinical efficacy of octreotide in the treatment of metastatic neuroendocrine tumors. A study by the Italian Trials in Medical Oncology Group, Cancer, 1996:77:402-8.

42. Ducreux M, Ruszniewski P, Chayvialle JA, et al., The antitumoral effect of the long-acting somatostatin analog lanreotide in neuroendocrine tumors, Am I Gastroenterol, 2000;95:3276-81.

43. Faiss S, Pape UF, Böhmig M, et al., Prospective, randomized, multicenter trial on the antiproliferative effect of lanreotide, interferon alfa, and their combination for therapy of metastatic neuroendocrine gastroenteropancreatic tumors - the International Lanreotide and Interferon Alfa Study Group, I Clin Oncol, 2003;21:2689-96.

44. Panzuto F, Di Fonzo M, lannicelli E, et al., Long-term clinical outcome of somatostatin analogues for treatment of progressive, metastatic, well-differentiated enteropancreatic endocrine carcinoma, Ann Oncol, 2006:17:461-6.

45. Saltz L, Trochanowski B, Buckley M, et al., Octreotide as an antineoplastic agent in the treatment of functional and nonfunctional neuroendocrine tumors, Cancer, 1993;72:244-8.

46. Wymenga AN, Eriksson B, Salmela PI, et al., Efficacy and safety of prolonged-release lanreotide in patients with gastrointestinal neuroendocrine tumors and hormonerelated symptoms, J Clin Oncol, 1999:17:1111.

47. Rinke A, Müller HH, Schade-Brittinger $\mathrm{C}$, et al., Placebocontrolled, double-blind, prospective, randomized study on the effect of octreotide LAR in the control of tumor growth in patients with metastatic neuroendocrine midgut tumors: a report from the PROMID Study Group, I Clin Oncol, 2009;27:4656-63.

48. Study of Lanreotide Autogel in Non-functioning Enteropancreatic Endocrine Tumours (CLARINET), ClinicalTrials.gov identifier NCT00353496. Available at:

www.clinicaltrials.gov/ct2/show/NCT00353496?term=NCTOO 353496\&rank=1 (accessed 9 July 2012).

49. Study of Pasireotide Long Acting Release (LAR) in Patients With Metastatic Neuroendocrine Tumors (NETS), ClinicalTrials.gov identifier NCT01253161, Available at: www.clinicaltrials.gov/ct2/show/NCT01253161?term=NCT01 253161\&rank=1 (accessed 9 July 2012)

50. Pavel ME, Hainsworth JD, Baudin E, et al., Everolimus plus octreotide long-acting repeatable for the treatment of advanced neuroendocrine tumours associated with carcinoid syndrome (RADIANT-2): a randomised, placebo-controlled, phase 3 study, Lancet, 2011;378:2005-12.

51. Oberg K, Kvols L, Caplin M, et al., Consensus report on the use of somatostatin analogs for the management of neuroendocrine tumors of the gastroenteropancreatic system, Ann Oncol, 2004;15:966-73. 